\title{
Effect of dephasing on charge-counting statistics in chaotic cavities
}

\author{
Ya.M. Blanter ${ }^{\mathrm{a}, \mathrm{b}, *}$, H. Schomerus ${ }^{\mathrm{c}}$, C.W.J. Beenakker ${ }^{\mathrm{c}}$ \\ 'Departement de Phystque Theorque Unversite de Geneve CH-1211 Geneve 4 Switzerland \\ ${ }^{\mathrm{b}}$ Department of Applied Physics Delft Unveisity of Teclnology Lorentzweg $12628 \mathrm{CJ}$ Delft The Netherlands \\ ${ }^{c}$ Instituut-Lorentz Untversiteit Leiden PO Bor 95062300 RA Lelden The Netherlands
}

Recelved 21 October 1999

\begin{abstract}
We calculate the cumulants of the charge transmitted through a chaotic cavity in the limit that the two openings have a large number of scattering channels The shot noise, which is the second cumulant, is known to be insensitive to dephasing in this limit Unexpectedly, the fourth and higher cumulants are found to depend on dephasing A semiclassical theory and a quantum mechanical model with strong dephasing give a result different from a fully phase-cohetent quantum mechanical theory (C) 2001 Elsevie1 Science B V All rights reserved
\end{abstract}

$P A C S \quad 0560-\mathrm{k}, 0365 \mathrm{Sq}, 7323 \mathrm{Ad}, 7350 \mathrm{Td}$

Keywords Shot norse, Fluctuations, Quantum statıstıcs, Transport processes, Quantum dots

\section{Introduction}

The theory of shot noise in mesoscopic systems has developed along two parallel lines, one fully quantum mechanical, the othei semiclassical (see Refs [1,2] for reviews) The semiclassical method treats the dynamics of the electrons according to classical mechanics, but includes quantum statistical effects following from

\footnotetext{
Presented at The International Workshop and Semmar on Dynamics of Complex Systems, Dresden, Germany, 31 Ma1ch-15 June 1999 See Physica E, Volume 9, 1ssue No 3

* Corresponding author Fax +31 15-278-1203
}

the Paulı exclusion principle The fully quantum mechanical method includes interference effects that are ignored in the semiclassical approach Both methods are expected to give identical results in the limit that the Fermı wavelength goes to zero, or equivalently, in the limit that the number of scattering channels $N$ goes to infinity, con esponding to a large conductance compared to the conductance quantum $e^{2} / h$

For example, in a diffusive conductor the shotnoise power $S \rightarrow \frac{2}{3} e\langle I\rangle$ in the limit $N \rightarrow \infty$ (with $\langle I\rangle$ the mean current), and this result has been obtained both quantum mechanically (using randommatrix theory [3] or Green's functions [4-6]) and 
semiclassically (using the Boltzmann-Langevin equation [7]). Another example of the correspondence principle between quantum mechanics and semiclassics in the large- $N$ limit is the shot noise of a double-barrier tunneling diode [8-11].

The shot-noise power is the second cumulant of the charge transmitted through the conductor in a certain time. For the complete counting statistics, one needs to know the higher cumulants as well. The only existing semiclassical calculation of higher cumulants was done for the double-barrier tunneling diode [11], and was found to be in complete agreement with the quantum mechanical theory [12-16] in the large- $N$ limit.

In this paper we present a semiclassical theory for the counting statistics of charge transported through a chaotic cavity, using the 'minimal correlation' approach developed recently by Sukhorukov and one of the authors [17]. Much to our surprise, we do not recover the quantum mechanical results in the large $-N$ limit: the fourth and higher cumulants differ. We need to introduce dephasing into the quantum mechanical theory (by means of a voltage probe [18-21]) to obtain agreement with semiclassics.

\section{Transmitted charge}

We consider a chaotic cavity (or quantum dot) connected to two equilibrium reservoirs by ballistic contacts (Fig. 1). (Since our results do not depend on the dimensionality of the cavity we focus on the two-dimensional situation, which simplifies notation.) A voltage $V$ is applied between the reservoirs. The reservoirs are described by equilibrium Fermi functions, which at zero temperature (the case of interest in this paper) have the form $f_{L}(E)=\theta(e V-E)$ and $f_{R}(E)=\theta(-E)$. The cross-sections of the contacts are denoted by $\Omega_{L}$ and $\Omega_{R}$. Since we want to exploit

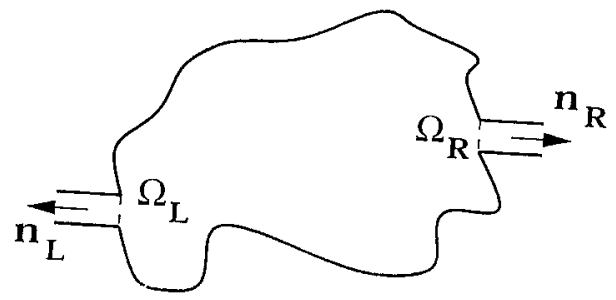

Fig. 1. Two-terminal chaotic cavity. The left and right contacts have cross-sections $\Omega_{L}, \Omega_{R}$, and outward normals $\mathbf{n}_{L}, \mathbf{n}_{R}$. the chaotic dynamics of the electron motion, we assume that there is no direct transmission between the contacts. In addition, the contacts are narrow as compared to the circumference of the cavity, but are classical in the sense that the number $N_{L}, N_{R}$ of propagating modes through each contact is large.

We are interested in the statistics of the charge transmitted through any of the contacts in a given time interval $t$,

$Q(t)=\int_{0}^{t} I\left(t^{\prime}\right) \mathrm{d} t^{\prime}$

where $I(t)=\langle I\rangle+\delta I(t)$ is the fluctuating current. We take the limit $t \rightarrow \infty$. For definiteness, we consider the current through the left contact, with the convention that positive current is directed towards the sample. In the long-time limit the charge transmitted through the right contact is the same, due to current conservation.

The average transmitted charge gives the average current $\langle I\rangle=\lim _{t \rightarrow \infty} t^{-1}\langle Q(t)\rangle$, and the variance of the transmitted charge gives the zero-frequency shot-noise power,

$$
\begin{aligned}
S & =2 \int_{-\infty}^{\infty}\langle\delta I(0) \delta I(t)\rangle \mathrm{d} t \\
& =2 \lim _{t \rightarrow \infty} t^{-1}\left(\left\langle Q^{2}\right\rangle-\langle Q\rangle^{2}\right) .
\end{aligned}
$$

Higher cumulants of $Q(t)$ determine higher-order correlation functions of the current.

Classically, the current is expressed in terms of the distribution function $f(\mathbf{r}, \mathbf{n}, E, t)$, with $\mathbf{n}$ the direction of momentum. The absolute value of the momentum $p_{\mathrm{F}}$ may be regarded as a constant. The current is given by

$$
\begin{aligned}
I_{L}(t)= & -\frac{e p_{\mathrm{F}}}{2 \pi \hbar^{2}} \int_{\Omega_{L}} \mathrm{~d} y_{L} \int \mathrm{d} E \\
& \times \int \mathrm{d} \mathbf{n}\left(\mathbf{n}_{L} \cdot \mathbf{n}\right) f(x, t),
\end{aligned}
$$

where $\mathbf{n}_{L}$ is the outward normal to the surface of the cavity at the location of the left contact (Fig. 1), $y_{L}$ is a point at the cross-section of the contact, and we have abbreviated $x \equiv\{\mathbf{r}, \mathbf{n}, E\}$. The angular integral is normalized to unity, $\int \mathrm{d} \mathbf{n}=1$.

Electrons incident from the left reservoir $\left(\mathbf{n}_{L} \cdot \mathbf{n}<0\right)$ are described by the non-fluctuating distribution function $f_{L}(E)$. (We ignore thermal fluctuations.) The distribution function of the electrons with $\mathbf{n}_{L} \cdot \mathbf{n}>0$ carries information about the chaotic dynamics inside 
the cavity On average, it only depends on energy, since the coordinate and angular dependence averages out due to the multıple scatterıng from the surface of the cavity This average distribution function inside the cavity is readily found from the condition that the current in each energy layer is conserved [17],

$$
\langle f\rangle=\frac{N_{L} f_{L}+N_{R} f_{R}}{N_{L}+N_{R}}
$$

The number of modes $N_{L}, N_{R}$ is given by $N_{L R}=$ $p_{\mathrm{F}} W_{L R} / \pi \hbar$, with $W_{L R}$ the width of the contacts The mean transmitted charge (1) is thus

$\langle Q\rangle=\left\langle I_{L}\right\rangle t=\frac{e^{2} V t}{h} \frac{N_{L} N_{R}}{N_{L}+N_{R}}$

This result could also be obtained by series addition of the ballistic conductances $N_{L} e^{2} / h$ and $N_{R} e^{2} / h$ of the two contacts

\section{Fluctuations of the distribution function}

Fluctuations of the transmitted charge at zero temperature are entirely determined by the fluctuations of the distribution function inside the cavity Following Ref [17], we assume that the fluctuations of the distribution function $\delta f \equiv f-\langle f\rangle$ inside the cavity may be decomposed into two parts,

$$
\delta f(x, t)=\delta \tilde{f}(x, t)+\delta f_{\mathrm{C}}(E, t)
$$

The function $\delta \tilde{f}$ describes the purely ballistic motion and obeys the equation

$$
\left(\partial_{t}+v_{\mathrm{F}} \mathbf{n} \nabla\right) \delta \tilde{f}(x, t)=0
$$

with $v_{\mathrm{F}}$ the Ferm1 velocity This equation is supplemented by the expression for the equal-time correlator,

$$
\left\langle\delta \tilde{f}(x, t) \delta \tilde{f}\left(x^{\prime}, t\right)\right\rangle=\delta\left(x-x^{\prime}\right)\langle f\rangle(1-\langle f\rangle),
$$

where

$$
\delta\left(x-x^{\prime}\right)=v^{1} \delta\left(\mathbf{r}-\mathbf{r}^{\prime}\right) \delta\left(\mathbf{n}-\mathbf{n}^{\prime}\right) \delta\left(E-E^{\prime}\right)
$$

and $v=m / 2 \pi \hbar^{2}$ is the density of states $\mathrm{Eq}$ (8) has the same form as in equilibrium, although the function $\langle f\rangle$ describes the non-equilibrium state in the cavity The reason is that the only source of norse at equilib11um as well as in the case of a deterministic chaotic cavity is the partial occupation of states The uniform fluctuating term $\delta f_{\mathrm{C}}$ accounts for the fact that at long times the motion inside the cavity is not purely ballistic, and ensures the current conservation at any time in any energy interval

The fluctuations in the current due to the term $\delta \tilde{f}$ are given by

$$
\begin{aligned}
& \delta \tilde{I}_{L}(t)=-\frac{e p_{\mathrm{F}}}{2 \pi \hbar^{2}} \int_{\Omega_{L}} \mathrm{~d} y_{L} \int \mathrm{d} E \\
& \times \int_{\left(\mathbf{n}_{L} \mathbf{n}\right)>0} \mathrm{dn}\left(\begin{array}{ll}
\mathbf{n}_{L} & \mathbf{n}) \delta \tilde{f}(x, t)
\end{array}\right.
\end{aligned}
$$

and similarly for $\delta \tilde{I}_{R}(t)$ The condition that the current at any time in any energy interval is conserved can be used to eliminate the fluctuation in the current due to the term $\delta f_{\mathrm{C}}$ The total fluctuation of the current is then given by

$\delta I_{L}(t)=\frac{N_{R} \delta \tilde{I}_{L}(t)-N_{L} \delta \tilde{I}_{R}(t)}{N_{L}+N_{R}}$

Eqs (6)-(8) constitute the minimal correlation approach, which was shown in Ref [17] to agree in the $\operatorname{limit} N_{L}, N_{R} \gg 1$ with the quantum mechanical expression for shot noise in a multi-terminal chaotic cavity We seek to extend this approach to higher cumulants of the transmitted charge Therefore, we have to specify all the cumulants of the function $\delta \tilde{f}$ at equal time moments Generalizing Eq (8), we write

$$
\begin{aligned}
& \left\langle\left\langle\delta \tilde{f}\left(x_{1}, t\right) \delta \tilde{f}\left(x_{2}, t\right) \quad \delta \tilde{f}\left(x_{k}, t\right)\right\rangle\right\rangle \\
& =\delta\left(x_{1}-x_{2}\right) \delta\left(x_{1}-x_{3}\right) \quad \delta\left(x_{1}-x_{k}\right) \\
& \quad \times\left\langle\left\langle f^{k}(x, t)\right\rangle\right\rangle,
\end{aligned}
$$

where $\langle\langle\rangle\rangle$ denotes the cumulant The cumulant $\left\langle\left\langle f^{k}(x, t)\right\rangle\right\rangle$ is calculated later on, it depends only on $E$, since moments of $f(x)$ are determined by $\langle f\rangle$ $\mathrm{Eq}$ (12) is equivalent to $\mathrm{Eq}(8)$ for $k=2$, since $\left\langle\left\langle f^{2}\right\rangle\right\rangle=\langle f\rangle(1-\langle f\rangle)$ (see below)

Taking into account Eq (7) and using Eq (12) as the initial condition, we obtain the expression for the cumulant of $\delta \tilde{f}$ at arbitrary times,

$$
\begin{aligned}
& \left\langle\left\langle\delta \tilde{f}\left(x_{1}, t_{1}\right) \delta \tilde{f}\left(x_{2}, t_{2}\right) \quad \delta \tilde{f}\left(x_{1}, t_{k}\right)\right\rangle\right\rangle \\
& =v^{1-1} \delta\left(E_{1}-E_{2}\right) \quad \delta\left(E_{k-1}-E_{k}\right) \\
& \times \delta\left(\mathbf{n}_{1}-\mathbf{n}_{2}\right) \quad \delta\left(\mathbf{n}_{k-1}-\mathbf{n}_{k}\right) \\
& \times \delta\left[\mathbf{r}_{1}-\mathbf{r}_{2}-v_{\mathrm{F}} \mathbf{n}_{\mathrm{I}}\left(t_{1}-t_{2}\right)\right] \quad \delta\left[\mathbf{r}_{k} \quad 1\right. \\
& \left.-\mathbf{r}_{t}-v_{\mathrm{F}} \mathbf{n}_{1}\left(t_{k} 1-t_{l}\right)\right]\left\langle\left\langle f^{\prime}\right\rangle\right\rangle
\end{aligned}
$$




\section{Semiclassical cumulants}

Eqs (3), (10), (11), and (13) may now be used to calculate the cumulants of the transmitted charge We note that all cumulants of the type $\left\langle\left\langle\delta \tilde{I}_{\alpha}\left(t_{1}\right) \quad \delta \tilde{I}_{\eta}\left(t_{k}\right)\right\rangle\right\rangle$ vanish due to the combination of delta functions in Eq (13), unless all the subscripts $\alpha, \quad, \eta$ are equal to $L$ or all are equal to $R$ The two non-zero cumulants are

$$
\begin{aligned}
& \left\langle\left\langle\delta \tilde{I}_{\alpha}\left(t_{1}\right) \delta \tilde{I}_{\alpha}\left(t_{2}\right) \quad \delta \tilde{I}_{\alpha}\left(t_{k}\right)\right\rangle\right\rangle \\
& =(-1)^{k} \frac{e^{k} N_{y}}{h} \int \mathrm{d} E\left\langle\left\langle f^{k}(E)\right\rangle\right\rangle \delta\left(t_{1}-t_{2}\right) \\
& \quad \times \delta\left(t_{k-1}-t_{k}\right), \quad \alpha=L, R, \quad k \geqslant 2
\end{aligned}
$$

Consequently, for the cumulant of the transmitted charge we obtain

$$
\begin{aligned}
\left\langle\left\langle Q^{k}\right\rangle\right\rangle= & \frac{e^{k} t}{h} \frac{N_{L}^{k} N_{R}+(-1)^{k} N_{R}^{k} N_{L}}{\left(N_{L}+N_{R}\right)^{k}} \\
& \times \int \mathrm{d} E\left\langle\left\langle f^{k}(E)\right\rangle\right\rangle
\end{aligned}
$$

To complete the calculation, we must compute the cumulant $\left\langle\left\langle f^{k}\right\rangle\right\rangle$ The fluctuating distribution function assumes only the values 0 and 1 , thus $\left\langle f^{k}\right\rangle=\langle f\rangle$ for $k \geqslant 1$ The characteristic function

$\chi(p)=\langle\exp (p f)\rangle=1+\langle f\rangle[\exp (p)-1]$

generates the cumulants as coefficients in a series expansion,

$\ln \chi(p)=\sum_{k-1}^{\infty} \frac{p^{k}}{k^{1}}\left\langle\left\langle f^{k}\right\rangle\right\rangle$

Substituting the average distribution function (4), integrating over energy, and taking into account Eq (5), we obtain the expression for the cumulants of the transmitted charge $(k \geqslant 2)$,

$\left\langle\left\langle Q^{k}\right\rangle\right\rangle=e^{k-1}\langle Q\rangle S_{k} \frac{N_{L}^{k-1}+(-1)^{k} N_{R}^{k-1}}{\left(N_{L}+N_{R}\right)^{k-1}}$,

where the coefficients $S_{k}$ are defined as

$\ln \left[N_{R}+N_{L} \exp (p)\right]=\sum_{k=0}^{\infty} \frac{p^{k}}{k^{!}} S_{k}$

Expression (18) may be simplified in the symmetric case $N_{L}=N_{R}$, when

$S_{2 l}=\frac{2^{2 l}-1}{2 l} B_{2 l}, \quad S_{2 l+1}=0, \quad l \geqslant 1$, where $B_{n}$ are Bernoull numbers In this case we have (for $l \geqslant 1$ )

$\left\langle\left\langle Q^{2 l}\right\rangle\right\rangle=e^{2 l-1}\langle Q\rangle \frac{2^{2 l}-1}{l 2^{2 l-1}} B_{2 l}$,

$\left\langle\left\langle Q^{2 l+1}\right\rangle\right\rangle=0$

In particular, $\left\langle\left\langle Q^{2}\right\rangle\right\rangle=e\langle Q\rangle / 4$, which is the $1 / 4$-shot noise suppression in a symmetric chaotic cavity [22] The next non-vanishing cumulant is negative, $\left\langle\left\langle Q^{4}\right\rangle\right\rangle=-e^{3}\langle Q\rangle / 32$

For non-equivalent contacts Eq (18) yrelds

$\left\langle\left\langle Q^{2}\right\rangle\right\rangle=e\langle Q\rangle \frac{N_{L} N_{R}}{\left(N_{L}+N_{R}\right)^{2}}$,

in agreement with Refs $[6,23]$ The next two cumulants are

$$
\begin{aligned}
\left\langle\left\langle Q^{3}\right\rangle\right\rangle= & -e^{2}\langle Q\rangle \frac{N_{L} N_{R}\left(N_{L}-N_{R}\right)^{2}}{\left(N_{L}+N_{R}\right)^{4}}, \\
\left\langle\left\langle Q^{4}\right\rangle\right\rangle= & e^{3}\langle Q\rangle N_{L} N_{R} \\
& \frac{\left(N_{L}^{2}-4 N_{L} N_{R}+N_{R}^{2}\right)\left(N_{L}^{3}+N_{R}^{3}\right)}{\left(N_{L}+N_{R}\right)^{7}}
\end{aligned}
$$

\section{Phase-coherent quantum mechanical cumulants}

We produced the result (18) by a semiclassical method, generalizing the minimal correlation approach to higher cumulants Let us compare it to the phase-coherent quantum mechanical theory in the large- $N$ limit

We use the relationshup derived by Lee et al [15] between the characteristic function of the transmitted charge and the transmission eigenvalues $T_{j}$,

$$
\langle\exp (Q p / e)\rangle=\prod_{,}\left[1+T_{J}(\exp (p)-1)\right]^{\mathrm{eVt} / \mathrm{h}}
$$

This is the expression for a particular cavity, so we still have to perform an ensemble average The transmission ergenvalues have density $\rho(T)$ in the ensemble of chaotic cavities The ensemble-averaged cumulants of the transmitted charge follow from

$$
\begin{aligned}
& \sum_{k-1}^{\infty} \frac{p^{k}}{k^{\prime}} e^{-k}\left\langle\left\langle Q^{k}\right\rangle\right\rangle \\
& \quad=\frac{e V t}{h} \int_{0}^{T} \mathrm{~d} T \rho(T) \ln [1+T(\exp (p)-1)]
\end{aligned}
$$


The density of transmission eigenvalues for $N_{L}$, $N_{R} \gg 1$ has the form $[6,23]$

$\rho(T)=\frac{\sqrt{N_{L} N_{R}}}{\pi T}\left(\frac{T}{1-T}-\frac{\left(N_{L}-N_{R}\right)^{2}}{4 N_{L} N_{R}}\right)^{1 / 2}$.

It vanishes for $T \leqslant\left[1+4 N_{L} N_{R}\left(N_{L}-N_{R}\right)^{-2}\right]^{-1}$.

In the symmetric case $N_{L}=N_{R}=N$ one simply has $[22,24]$

$\rho(T)=\frac{N}{\pi} \frac{1}{\sqrt{T(1-T)}}$

and hence

$\sum_{k=1}^{\infty} \frac{p^{k}}{k !} e^{1-k}\left\langle\left\langle Q^{k}\right\rangle\right\rangle=4\langle Q\rangle \ln \left[\frac{1}{2}+\frac{1}{2} \exp (p / 2)\right]$,

which is equivalent to Eqs. (18) and (20). Thus, for the symmetric case the results for the charge counting statistics, obtained semiclassically and quantum mechanically, are identical.

This correspondence does not extend to the more general case $N_{L} \neq N_{R}$. From Eqs. (26) and (27) we reproduce the semiclassical expressions (22) and (23) for $\left\langle\left\langle Q^{2}\right\rangle\right\rangle$ and $\left\langle\left\langle Q^{3}\right\rangle\right\rangle$. However, the fourth cumulant,

$$
\begin{aligned}
\left\langle\left\langle Q^{4}\right\rangle\right\rangle= & e^{3}\langle Q\rangle N_{L} N_{R}\left(N_{L}+N_{R}\right)^{-6} \\
& \times\left(N_{L}^{4}-8 N_{L}^{3} N_{R}+12 N_{L}^{2} N_{R}^{2}-8 N_{L} N_{R}^{3}+N_{R}^{4}\right),
\end{aligned}
$$

is different from Eq. (24).

\section{Effect of dephasing on the quantum mechanical cumulants}

Apparently, the difference between Eqs. (24) and (30) is due to the lack of phase coherence in the semiclassical minimal correlation approach. To strengthen this explanation, we incorporate into the quantum mechanical theory a dephasing mechanism via an additional fictitious lead attached to the cavity (see Fig. 2). Following Refs. [20,21] we assume that this lead has a (fluctuating) distribution function which is determined from the condition that no current flows into the reservoir at every energy and every instant of time. An electron absorbed by this lead is immediately reinjected back at the same energy, without any memory of the phase. Thus, this fictitious lead

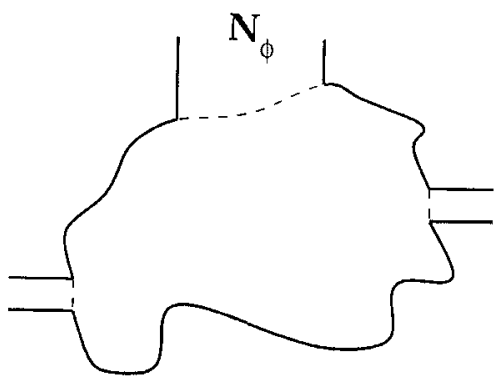

Fig 2 Chaotic cavity with a fictitious lead (supporting $N_{\phi}$ propagatung modes) that models strong uniform dephasing.

introduces dephasing but not inelastic scattering. For homogeneous and complete dephasing, we take the number of scattering channels $N_{\phi}$ in the dephasing lead to be very large, $N_{\phi} \gg N_{L}, N_{R}$.

Our starting point is the expression for the current operator in the lead $\alpha(\alpha=\mathrm{L}, \mathrm{R}, \phi)$ in terms of creation/annihilation operators $[8,2]$,

$$
\begin{aligned}
\hat{I}_{\alpha}(t)= & \frac{e}{h} \sum_{\gamma \delta} \sum_{m n} \int \mathrm{d} E \int \mathrm{d} E^{\prime} \exp \left(\frac{\mathrm{i}\left(E-E^{\prime}\right) t}{\hbar}\right) \\
& \times \hat{a}_{\gamma m}^{\dagger}(E) A_{\gamma \delta}^{m n}\left(\alpha, E, E^{\prime}\right) \hat{a}_{\delta n}\left(E^{\prime}\right) .
\end{aligned}
$$

The sum over $\gamma$ and $\delta$ is over the leads $L, R$, and $\phi$, the sum over $m, n$ is over the mode indices. The current matrix $A$ is expressed in terms of the scattering matrices $s$,

$A_{\gamma \delta}^{m n}\left(\alpha, E, E^{\prime}\right)=\delta_{m n} \delta_{\alpha \gamma} \delta_{\alpha \delta}-s_{\alpha \gamma}^{\dagger m n}(E) s_{\alpha \delta}^{m m}\left(E^{\prime}\right)$

and the creation and annihilation operators obey the fermion anticommutation relation

$$
\begin{aligned}
& \hat{a}_{\alpha m}^{\dagger}(E) \hat{a}_{\beta n}\left(E^{\prime}\right)+\hat{a}_{\beta n}\left(E^{\prime}\right) \hat{a}_{\alpha m}^{\dagger}(E) \\
& \quad=\delta_{\alpha \beta} \delta_{m n} \delta\left(E-E^{\prime}\right) .
\end{aligned}
$$

The expectation value

$\left\langle a_{\alpha m}^{\dagger}(E) \hat{a}_{\beta n}\left(E^{\prime}\right)\right\rangle=\delta_{\alpha \beta} \delta_{m n} \delta\left(E-E^{\prime}\right) f_{\alpha}(E)$

is given by the average distribution function $f_{\alpha}(E)$ in reservoir $\alpha$. From the condition that the average (energy resolved) current $\left\langle I_{\phi}(E)\right\rangle$ through the dephasing lead vanishes at every energy, one finds the average distribution function

$f_{\phi}(E)=\frac{N_{L} f_{L}+N_{R} f_{R}}{N_{L}+N_{R}}$,

which is identical to the average semiclassical distribution function (4) inside the cavity. 
The distribution function of the dephasing lead fluctuates in time These fluctuations, which can also be found from the condition that the current through this lead vanishes, modify the fluctuations of the current at the leads $L$ and $R$ [21],

$\Delta \hat{I}_{L}(t)=\delta \hat{I}_{L}(t)+\frac{N_{L}}{N_{L}+N_{R}} \delta \hat{I}_{\phi}(t)$,

$\Delta \hat{I}_{R}(t)=\delta \hat{I}_{R}(t)+\frac{N_{R}}{N_{L}+N_{R}} \delta \hat{I}_{\phi}(t)$,

where the intrinsic fluctuations $\delta \hat{I}_{\alpha}(t)=\hat{I}_{\alpha}(t)-\left\langle\hat{I}_{\alpha}(t)\right\rangle$ are described by Eq (31)

We now calculate the fourth cumulant of the charge transmitted through the left lead,

$\left\langle\left\langle Q^{4}\right\rangle\right\rangle=\left\langle\left\langle\left(\int_{0}^{t} \mathrm{~d} t^{\prime} \Delta \hat{I}_{L}\left(t^{\prime}\right)\right)^{4}\right\rangle\right\rangle$

Explicitly, we have

$$
\begin{aligned}
\left\langle\left\langle Q^{4}\right\rangle\right\rangle= & \Xi_{L L L L}+\frac{4 N_{L}}{N_{L}+N_{R}} \Xi_{L L L \phi} \\
& +\frac{6 N_{L}^{2}}{\left(N_{L}+N_{R}\right)^{2}} \Xi_{L L \phi \phi}+\frac{4 N_{L}^{3}}{\left(N_{L}+N_{R}\right)^{3}} \Xi_{L \phi \phi \phi} \\
& +\frac{N_{L}^{4}}{\left(N_{L}+N_{R}\right)^{4}} \Xi_{\phi \phi \phi \phi}
\end{aligned}
$$

where we have defined the cumulant

$\Xi_{\sigma_{1} \alpha_{2} \alpha_{3} \alpha_{4}}=\left\langle\left\langle\prod_{l=1}^{4} \int \mathrm{d} t_{l} \hat{I}_{\alpha}\left(t_{l}\right)\right\rangle\right\rangle$

in terms of the current operators (31) It remains to calculate the cumulant of eight creation/annihilation operators, by taking into account all possible pair1ngs between the operators $\hat{a}_{j m}^{\dagger}$ and $\hat{a}_{\delta n_{i}}(l=1,2,3,4)$ which couple all the four current operators together For $N_{\phi} \gg N_{L}, N_{R} \gg 1$ the leading contribution to the cumulant comes from the terms with $\gamma_{t}=\delta_{t}=\phi$ Summing all possible parrings, we obtain

$$
\begin{aligned}
\Xi_{\alpha_{1} \alpha_{2} \alpha_{3} \alpha_{4}}= & \frac{e^{4} t}{h} \operatorname{Tr}\left[A_{\phi \phi}\left(\alpha_{1}\right) \quad A_{\phi \phi}\left(\alpha_{4}\right)\right] \int \mathrm{d} E \\
& \times f_{\phi}\left(1-f_{\phi}\right)\left[f_{\phi}^{2}-4 f_{\phi}\left(1-f_{\phi}\right)\right. \\
& \left.+\left(1-f_{\phi}\right)^{2}\right],
\end{aligned}
$$

where the trace 1 s taken over the mode indices In the same leading order, we neglect all traces of the type $\operatorname{Tr}\left[s_{\alpha \phi}^{\dagger} \quad s_{\eta \phi}\right](\alpha \quad \eta=L, R)$, unless all indices $\alpha \quad \eta$ are the same In this case

$\operatorname{Tr}\left[s_{L \phi}^{\dagger} s_{L \phi}\right]^{4}=N_{L}, \quad \operatorname{Tr}\left[s_{R \phi}^{\dagger} s_{R \phi}\right]^{4}=N_{R}$
Summing all contributions in Eq (37), we recover the result (24) of the semiclassical theory

\section{Conclusions}

To summarize, we have studied the charge counting statistics in chaotic cavities using three different approaches (1) a fully coherent quantum mechanical theory, (11) a dephasing-lead model that is also quantum mechanical but phenomenologically introduces uniform and strong dephasing inside the cavity, and (111) the semiclassical minimal correlation approach All three approaches give the same results if the two openings in the cavity have the same (large) number of scattering channels The cumulants for this symmetric case are given by Eq (21) In the asymmetric case of two different openings, the first three cumulants are also the same in the three approaches, but the fourth cumulant is not For approach (1) it is given by $\mathrm{Eq}$ (30), for approaches (11) and (111) by Eq (24)

We conclude that the counting statistics of a chaotic cavity is sensitive to dephasing even in the limit of a large number of scattering channels This is an unexpected conclusion since the shot noise is not affected by dephasing What is lacking is an understanding in physical terms as to why the high-order cumulants of the transmitted charge respond differently to dephasing than the low-order cumulants

\section{Acknowledgements}

We thank the Max-Planck-Institut fur Physık Komplexer Systeme in Dresden for hospitality and support We acknowledge support by the Sw1ss National Sc1ence Foundation via the Nanoscience program, by the Dutch Science Foundation NWO/FOM, and by the TMR program of the European Commission

\section{References}

[1] M J M de Jong, CW J Beenakker, in L L Sohn, L P Kouwenhoven, G Schon (Eds), Mesoscopic Election Transport, NATO ASI Series E345, Kluwe1, Do1diecht, 1997, p 225

[2] Ya M Blanter, M Buttıke, Phys Rep 336 (2000) 1

[3] C W J Beenakker, M Buttıker Phys Rev B 46 (1992) 1889 
[4] B L Altshuler, L S Levitov, A Yu Yakovets, Pis'ma Zh Eksp Teor Fiz 59 (1994) 821 (JETP Lett 59 (1994) 857)

[5] Yu V Nazarov, Phys Rev Lett 73 (1994) 134

[6] Yu V Nazarov, in H A Ceidexra, B Kuamer, G Schon (Eds), Quantum Dynamics of Submicion Structures, NATO ASI Ser1es E291, Kluwer, Dordrecht, 1995, p 687

[7] KE Nagaev, Phys Lett A 169 (1992) 103

[8] M Buttiker, Physica B 175 (1991) 199

[9] L Y Chen, C S Ting, Phys Rev B 43 (1991) 4534

[10] J H Davies, P Hyldgaard, S Hershfield, J W Wilkms, Phys Rev B 46 (1992) 9620

[11] M J M de Jong, Phys Rev B 54 (1996) 8144

[12] L S Levitov, GB Lesovik, P1s'ma Zh Eksp Teor Fiz 58 (1993) 225 (JETP Lett 58 (1993) 230)

[13] D A Ivanov, L S Levitov, P1s'ma Zh Eksp Teor F1z 58 (1993) 450 (JETP Lett 58 (1993) 461)

[14] B A Muzykantskı1, DE Khmelnıtskı1, Phys Rev B 50 (1994) 3982
[15] H Lee, L S Levitov, A Yu Yakovets, Phys Rev B 51 (1995) 4079

[16] L S Levitov, H Lee, G B Lesovik, J Math Phys 37 (1996) 4845

[17] Ya M Blanter, EV Sukhorukov, Phys Rev Lett 84 (2000) 1280

[18] M Buttıkeı, Phys Rev B 33 (1986) 3020

[19] M Buttiker, IBM J Res Dev 32 (1988) 63

[20] M J M de Jong, C W J Beenakker, Physica A 230 (1996) 219

[21] S A van Langen, M Buttker, Phys Rev B 56 (1997) R1680

[22] R A Jalabert, J -L Pichard, C W T Beenakker, Europhys Lett 27 (1994) 255

[23] C W J Beenakker, Rev Mod Phys 69 (1997) 731

[24] H U Baranger, P Mello, Phys Rev Lett 73 (1994) 142 